\title{
Implementing malaria control in South Africa, Eswatini and southern Mozambique during the COVID-19 pandemic
}

\author{
B D Brooke, ${ }^{1,2} \mathrm{PhD}$; J Raman, ${ }^{1,2} \mathrm{PhD}$; J Frean, ${ }^{1,2} \mathrm{MB}$ ChB, MMed (Path Microbiol); K Rundle, ${ }^{3}$ BIS Publishing; \\ F Maartens, ${ }^{3}$ MSc; E Misiani, ${ }^{4} \mathrm{PhD}$, MPH; A Mabuza, ${ }^{5}$ National Diploma in Public Health; \\ K I Barnes, ${ }^{6,7}$ MB ChB, MMed (Clinical Pharmacology); D P Moonasar, ${ }^{4,8}$ DrPH; Q Dlamini, ${ }^{9}$ PGDip (DAP\&E); \\ S Charles, ${ }^{3}$ BAcc, CASA; L Blumberg, ${ }^{1,10}$ MB BCh, MMed (Micro), DTM\&H, DOH, DCH \\ ${ }^{1}$ Centre for Emerging Zoonotic and Parasitic Diseases, National Institute for Communicable Diseases, National Health Laboratory Service, \\ Johannesburg, South Africa \\ ${ }^{2}$ Wits Research Institute for Malaria, School of Pathology, Faculty of Health Sciences, University of the Witwatersrand, Johannesburg, South Africa \\ ${ }^{3}$ Lubombo Spatial Development Initiative and Goodbye Malaria, Johannesburg, South Africa \\ ${ }^{4}$ Malaria and Other Vector-Borne and Zoonotic Diseases Directorate, National Department of Health, Pretoria, South Africa \\ ${ }^{5}$ Malaria Programme, Clinton Health Access Initiative, Pretoria, South Africa \\ ${ }^{6}$ Division of Clinical Pharmacology, Department of Medicine, Faculty of Health Sciences, University of Cape Town, South Africa \\ ${ }^{7}$ WorldWide Antimalarial Resistance Network (WWARN), Division of Pharmacology, Faculty of Health Sciences, University of Cape Town, \\ South Africa \\ ${ }^{8}$ School of Health Systems and Public Health, Faculty of Health Sciences, University of Pretoria, South Africa \\ ${ }^{9}$ National Malaria Control Programme, Mbabane, Kingdom of Eswatini \\ ${ }^{10}$ Faculty of Veterinary Science, University of Pretoria, South Africa
}

Corresponding author: L Blumberg (lucilleb@nicd.ac.za)

The COVID-19 pandemic has strained healthcare delivery systems in a number of southern African countries. Despite this, it is imperative that malaria control and elimination activities continue, especially to reduce as far as possible the number and rate of hospitalisations caused by malaria. The implementation of enhanced malaria control/elimination activities in the context of COVID-19 requires measures to protect healthcare workers and the communities they serve. The aim of this review is therefore to present innovative ideas for the timely implementation of malaria control without increasing the risk of COVID-19 to healthcare workers and communities. Specific recommendations for parasite and vector surveillance, diagnosis, case management, mosquito vector control and community outreach and sensitisation are given.

S Afr Med J 2020;110(11):1072-1076. https://doi.org/10.7196/SAMJ.2020.v110i11.15286

The COVID-19 global pandemic reached South Africa (SA), Mozambique and Eswatini in March 2020. ${ }^{[1]}$ Since then an exponential increase in SARS-CoV-2 infections has severely stretched SA's healthcare system, especially in terms of in-hospital treatment of severe cases. The impact of COVID-19 in Mozambique and Eswatini at the time of writing has been comparatively mild, but is increasing. It is therefore imperative to reduce as far as possible the number and rate of hospitalisations caused by trauma and other diseases, including malaria.

Malaria incidence in SA is seasonal and peaks in the wetter summer months, especially during January to April. ${ }^{[2]}$ Although malaria incidence in SA is currently low, the risk of outbreaks is always present, with the most recent having occurred in 2017 and, at a more localised level in Limpopo Province, in 2019. The reasons for these latest outbreaks are varied and include unusually high rainfall and cross-border movement of migrant populations, fuelling local transmission. These issues are particularly pertinent to COVID-19 in SA's malaria-affected districts. They highlight the importance of mitigating factors contributing to high malaria incidence and consequent hospitalisations, which may be further exacerbated by COVID-19/malaria coinfections and the re-opening of SA's borders with those neighbouring countries with higher malaria transmission intensities.

Locally acquired malaria in SA primarily occurs in the three endemic provinces of Limpopo, Mpumalanga and KwaZulu-Natal. This is due to the perennial presence of malaria-transmitting Anopheles mosquito populations in these areas. Other provinces, especially Gauteng, report and treat comparatively high numbers of imported cases, mainly from Mozambique. SA's long history of effective malaria control has led to a low incidence, making elimination a feasible prospect, as detailed in the country's malaria strategic plan for the period $2019-2023 .{ }^{[3]}$ The main interventions at the provincial level include mosquito vector control by indoor residual spraying (IRS) of specially formulated insecticides, larval source management, and prompt diagnosis and effective treatment of all confirmed malaria cases. The effectiveness of these interventions depends on enhanced active and passive case detection (parasite surveillance), intensive mosquito vector surveillance, community outreach programmes, and carefully co-ordinated management of these activities at national and provincial levels, with support from partner institutions in terms of specialised diagnostics, operational research and training. 
Malaria incidence in the Kingdom of Eswatini is low and the country is therefore in a strong position to eliminate malaria in the near future. ${ }^{[4]}$ As most cases in Eswatini are imported, with very little local transmission, case-based surveillance has been substantially strengthened under the MOSASWA cross-border initiative. MOSASWA (Mozambique, South Africa and Eswatini) is a sub-regional collaborative initiative based on evidence that malaria is a regional problem in southern Africa. These three countries are working collaboratively to move SA and Eswatini to elimination and southern Mozambique to pre-elimination, with MOSASWA supporting catalytic activities at sub-regional and in-country transmission areas. ${ }^{[5]}$

Mozambique is holoendemic for malaria and accounts for $46 \%$ of the malaria burden in southern Africa, averaging 9.3 million cases per year. ${ }^{[0,7]}$ A regional malaria elimination initiative, supported by Goodbye Malaria, the Global Fund for HIV, Tuberculosis and Malaria, the governments of Mozambique, SA and Eswatini (MOSASWA), the Bill and Melinda Gates Foundation and private sector partners, and implemented by the Lubombo Spatial Development Initiative 2, is currently operational. The core intervention of this initiative is IRS in Maputo, Gaza and Inhambane provinces of southern Mozambique.

The current COVID-19 pandemic has disrupted regional, national and provincial malaria control programmes in general and has made planning and preparation slower and more complicated. Implementing and sustaining these enhanced malaria control/ elimination activities in the context of COVID-19 requires innovative measures to protect healthcare workers and the communities they serve. The objective of this review is to present innovative ideas for the timely implementation of malaria control without increasing the risk of COVID-19 among healthcare workers and the malariaaffected communities.

\section{Case detection and parasite surveillance}

Accurate current intelligence on malaria parasites and incidence has become even more crucial in the face of the COVID-19 pandemic. Any interruption in routine surveillance and control activities risks malaria rebounding with severe consequences, as seen in the recent West African Ebola outbreaks. ${ }^{[8]}$ As COVID-19 control measures, such as physical distancing and restricted movements of people, have the potential to limit routine surveillance activities, malaria surveillance systems must be flexible enough to adopt and implement innovative strategies that enable the collection and analysis of essential data to continue.

With the provincial malaria control programmes forced to downscale their active case-finding activities, community healthcare workers, particularly those in border communities frequented by mobile and migrant populations, should be supported and capacitated to fill this vital surveillance gap. Community healthcare workers need to be adequately trained and equipped with the required personal protective equipment (PPE), testing devices and necessary casereporting tools in order to assume this responsibility. As mobile applications for immediate notification, such as MalariaConnect ${ }^{[9]}$ and the Notifiable Medical Conditions App, are readily available, there should be no delays in the notification of cases.

Since community healthcare workers are not currently permitted to treat patients for malaria, a referral system that allows patients with uncomplicated malaria to receive treatment, and those with danger signs or severe malaria to be transported to the appropriate healthcare facility, is needed. Community healthcare workers could also play a critical role in sourcing details required for accurate case classification. In addition, they could monitor and document patient adherence and treatment response. This would help the provincial malaria control programmes achieve higher and quicker coverage with case investigations and patient follow-up, and should be done telephonically if home visits are not possible.

\section{Mallaria diagnosis}

In SA, given its low malaria prevalence and limited extent of active surveillance, most malaria cases are detected on symptomatic presentation, predominantly in public sector clinics and hospitals. The early clinical presentation is one of acute undifferentiated febrile illness. Many other infections, which now include COVID-19, present in a similar way. ${ }^{[10]}$ Fever, chills, fatigue, headache, and joint and muscle pain are clinical features common to the early stages of both malaria and COVID-19, as well as numerous other infections. Delayed health seeking for malaria is common in SA, and the more severe presentations include respiratory distress and metabolic acidosis, which could be confused with COVID-19 pneumonia. $\mathrm{X}$-rays could potentially indicate the more likely diagnosis, but a radiology service is not always readily available. Point-of-care tests (rapid diagnostic tests, RDTs) and/or blood film microscopy are relatively quick (taking 15 - 20 minutes for the former and an hour for the latter) and widely available methods for malaria diagnosis, whereas definitive detection of SARS-CoV-2 requires polymerase chain reaction technology that is less readily available, with longer delays (up to several days) before results are available. There should therefore be a low threshold for testing for malaria in transmission areas (and wherever imported malaria is common, such as Gauteng) to definitively diagnose or exclude malaria as the cause of illness. For blood sampling, in addition to gloves, COVID-19-related PPE for healthcare workers is mandatory. Some ancillary laboratory investigations may give early clues to the diagnosis, e.g. malaria patients often have thrombocytopenia, whereas lymphopenia is a feature of COVID-19. High bilirubin levels and jaundice are typical of severe malaria, but are much less commonly seen in COVID-19. Given the challenges of providing large-scale molecular testing for resource-restricted African countries, ${ }^{[11]}$ the preliminary report of a SARS-CoV-2 antigen detection RDT with a diagnostic accuracy of $96 \%$ is potentially promising, but larger studies are required..$^{[12]}$

'Stay at home' orders, curfews, reduced public transport, other COVID-19 lockdown restrictions and fear of COVID-19 exposure at health facilities all have the potential to impede health-seeking behaviour, which could delay diagnosis of malaria and other potentially serious infections such as meningitis and bacterial pneumonia, as well as COVID-19. ${ }^{[13]}$ Reduced opportunities for travel to transmission areas (e.g. Mozambique, Zimbabwe) may also decrease the number of imported malaria cases, which can reduce healthcare worker awareness of malaria in the face of the COVID19 pandemic. As the malaria transmission season (October to May) commences, the usual malaria alerts and reminders targeting healthcare workers and the public assume even greater importance, and need to emphasise the overlap with COVID-19. It is clearly possible for both pathogens to infect an individual - simultaneous malaria and COVID-19 infection has been described ${ }^{[14]}-$ but this is more likely to occur in high-transmission malaria areas.

\section{Case management}

In non-immune individuals (SA residents are non-immune, including residents in malaria transmission areas in SA, because malaria transmission intensity here is too low and seasonal for acquiring partial malaria immunity), malaria progresses more rapidly to severe 
disease, usually requiring intensive care. Early, effective malaria treatment is therefore essential for curing malaria and to reduce the burden on the healthcare system. Personal safety concerns and COVID-19-related movement restrictions described above have exacerbated late treatment-seeking behaviour. Healthcare workers must facilitate the early diagnosis and prompt, effective management of malaria by maintaining a high index of suspicion for malaria, testing every acutely ill, febrile patient who resides in, or has visited, a malaria area.

Artemisinin-based therapies remain the most effective treatment for malaria in SA and globally. These recommended treatments, artemether-lumefantrine for uncomplicated malaria and injectable artesunate for severe malaria, must be readily available at public and private health facilities. Severe malaria is a medical emergency, and treatment with intravenous artesunate must be started as soon as the diagnosis is made. Compared with intravenous quinine, intravenous artesunate is associated with significantly better treatment outcomes, ${ }^{[15]}$ with malaria deaths decreased by $39 \%$ in adults and $24 \%$ in children, ${ }^{[16]}$ a better safety profile, a simpler dosing regimen and increased cost-effectiveness. As the demand for intensive care beds is high as a result of COVID-19, it is essential that hospitals stock intravenous artesunate and treat severe malaria with it to reduce the demands on critical care beds and healthcare workers. Patients with uncomplicated malaria can be safely and effectively treated with artemether-lumefantrine as outpatients, after excluding repeated vomiting, danger signs and features of severe malaria. Patients should complete the full 6-dose course over 3 days, even if they feel better earlier. Doses should ideally be taken with a fat-containing food or drink to aid absorption of the antimalarials. Patients should be advised to return to the health facility promptly if doses are vomited or they deteriorate in any way. High-risk groups, including pregnant women, infants and those with significant comorbidities such as malnutrition, may require closer monitoring of treatment response (ideally daily) if treated as outpatients.

At present there is no clinical evidence that artemisinin or its derivatives are effective in either treating or preventing COVID19. Any off-label use of artemisinin products should therefore be avoided, to avoid drug resistance and preserve stocks of these life-saving antimalarials. To prevent or slow the development of resistance to artemisinin and its derivatives, the World Health Organization (WHO) strongly recommends that these therapies be reserved solely for the treatment of malaria. The repurposing of two older antimalarials, chloroquine and hydroxychloroquine, for the treatment of COVID-19 has been suggested. However, clinical trial data to date have shown that they are not effective for treating mild or severe COVID-19 infections ${ }^{[17]}$ or as prophylaxis, ${ }^{[18]}$ and that they are associated with potentially severe adverse effects ${ }^{[19]}$ such as a prolonged QT interval. ${ }^{[20]}$ These antimalarials should therefore not be used for the treatment or prevention of COVID-19 infections outside the context of clinical trials.

\section{Mosquilto vector control}

Of the vector control interventions implemented by SA's provincial malaria programmes, IRS poses the highest risk of exposure to COVID-19. This is because the IRS spray teams deliver a community service that increases their risk of COVID-19 exposure. The increased risk is due to direct person contact (e.g. at training meetings, household visits for IRS application, daily meetings with supervisors and other team members) and contact with potentially contaminated surfaces (e.g. door handles, furniture and spray pump equipment). This increased risk can result in psychological distress, work fatigue and stigma. It is therefore essential that team leaders and spray operators receive additional training regarding activities that impose risks and how to mitigate them effectively. Good work practices designed to mitigate the risks of COVID-19 will protect both the spray team members and the communities they serve (examples described below). An integrated approach between the malaria surveillance teams and the district/subdistrict infection prevention and control teams is already in place, and this approach should work for the IRS teams as well.

\section{Cost of IRS implementation}

In order to accommodate COVID-19 regulations, IRS operations conducted during the pandemic will be more expensive than usual. Additional resources to cover this gap should be made available, and funding for malaria control should not be redirected to fight COVID19 , given that malaria is also a life-threatening disease.

\section{Pre-intervention planning and procurement}

Planning for seasonal IRS is an especially important step that ultimately determines the success of each spray round. Advanced IRS planning is necessary to mitigate unforeseen delays. Typical delays caused by the COVID-19 pandemic include increased time to delivery of products and consumables due to closed and/or slow border gates, slower offloading of shipping containers at harbours due to minimal available staff and shortages of PPE, and the prioritising of consumables and equipment for the management of COVID-19 by supply chain managers. It is therefore critical that malaria control programmes order their spraying equipment, PPE and insecticide much earlier than usual to accommodate these delays. Provincial programmes should also consider increasing their amounts of buffer stock to mitigate delays in procurement going forward.

IRS planning needs to ensure alignment with local and international COVID-19 prevention guidelines so that subsequent IRS training and operations are conducted in a safe and responsible manner. For example, the transportation of spray operators and other malaria control workers must adhere to local lockdown regulations.

\section{IRS team recruitment and training}

During the COVID-19 pandemic, the recruitment procedure for spray operators should give preference to those with previous experience so as to reduce the number of training days required. Age and health profiling should be taken into consideration during this process, as spray operators aged $>55$ years and those with comorbidities are at elevated COVID-19 risk.

IRS training during the COVID-19 pandemic also requires a different approach, owing to restrictions on the movement of people and interprovincial/district travel. An additional complication is the restriction on the number of people that may gather. This affects IRS training events at large centralised training centres. It is therefore necessary to extend the training period and budget to allow for the gathering of smaller groups (e.g. 50 spray operators per session, depending on the training area available). This can be done by identifying localised training centres (one per district/subdistrict) for smaller groups. Each centre should have ablution facilities, running water, and walls that can be used for practical IRS training. During training (and subsequent IRS operations), symptom screening of spray team members should be conducted daily, and the wearing of masks, hand sanitising and physical distancing should be monitored and enforced at all times.

As a consequence of movement restrictions in southern Mozambique, a creative and innovative solution was required to 
facilitate spray operator training in each district. This involved the design and construction of mobile training walls (Fig. 1). These are made from waterproofed wood frames and marine ply sheets. A set of these walls will be distributed to each district, where they will be erected on the sides of the storage facilities and/or against the wash facility fence line for IRS training purposes. These walls are crucial for IRS spray operator training, as they enable the trainees to master the insecticide application technique (i.e. the distance between nozzle and wall surface, and the direction and speed of movement when spraying), which in turn determines the efficacy of the insecticide application and ultimately its impact on malaria transmission. The innovative method of conducting district-level training using mobile wall kits may revolutionise IRS training and malaria control in future.

\section{IRS operations}

IRS coverage should be stratified according to malaria risk based on its incidence in previous seasons. ${ }^{[3]}$ The highest-risk areas should be prioritised for spraying, followed by lower-risk areas, all of which can be additionally protected by treating known Anopheles mosquito breeding sites with an appropriate larvicide. IRS and larval control activities should be conducted especially timeously (early September to December) and efficiently, and spray teams should furnish the communities they serve with malaria and COVID-19 prevention messages to reassure community members that they are not bringing COVID-19 infection into their homes as they move from house to house during the spray campaign. Spray team members should practise physical distancing and wear full PPE at all times, even in vehicles, with frequently touched surfaces and equipment (including mobile phones and tablets) regularly disinfected.

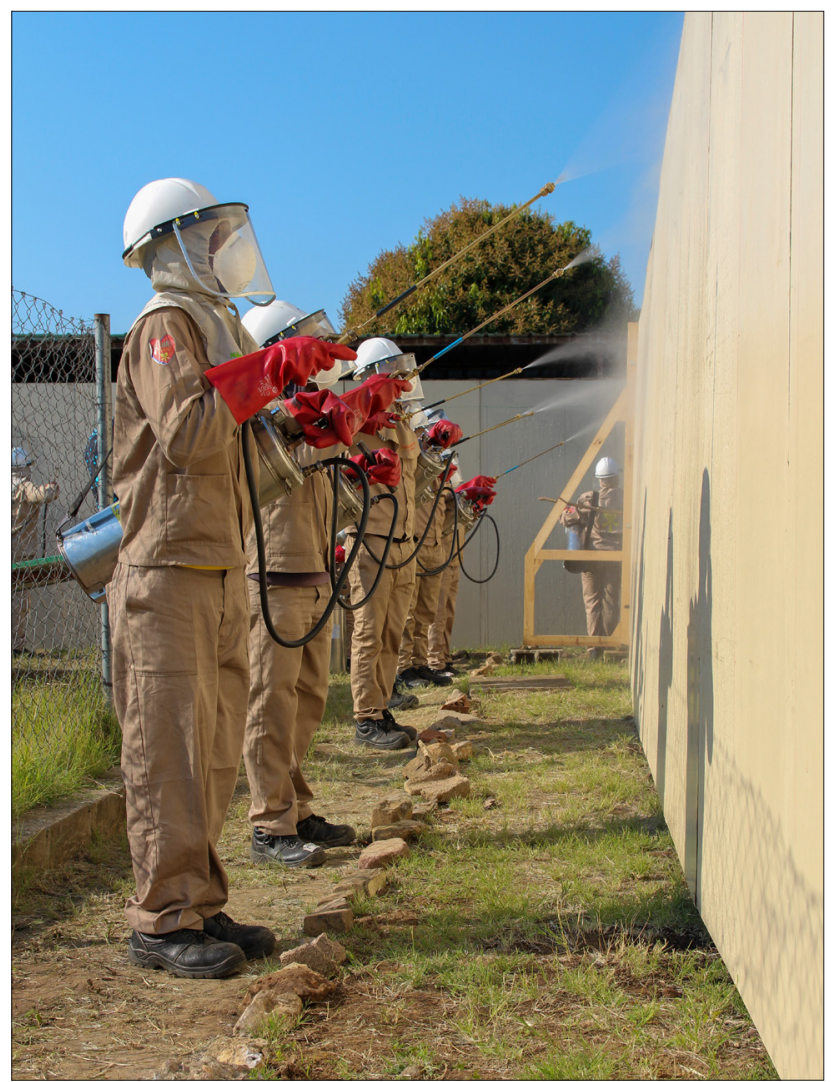

Fig. 1. Mobile wall units used for the training of spray operators in southern Mozambique.

\section{Vector surveillance}

Ensuring that entomological surveillance continues during the COVID-19 pandemic is particularly important. This is because essential surveillance indicators are needed by the province and district/municipality on a regular basis to inform control strategies such as insecticide choice for IRS operations. ${ }^{[3]}$ The important entomological indicators required include vector species assemblages by region and season, relative vector densities, insecticide susceptibilities, and the breeding, biting and resting behaviours of each incriminated species. ${ }^{[21]}$

Most of the mosquito collection/sampling techniques currently used in SA's malaria-endemic provinces are based on outdoor collections. ${ }^{[22]}$ This is because the IRS insecticiding programmes have made finding indoor-resting mosquitoes especially difficult. These outdoor collection methods include the use of natural and artificial resting shelters (such as pit traps, ceramic pots and tyres), human landing catches, bait-net traps, animal shelter/kraal searches and larval collections. These are appropriate for use during the COVID19 pandemic because they are conducted outdoors in well-ventilated spaces, they enable appropriate physical distancing, and house entry by entomology team members is not necessary. In instances where the collection of indoor-resting mosquitoes is indicated, such as the monitoring of indoor-resting mosquitoes in sprayed dwellings, exit window traps, pyrethroid spray catches (PSCs) and/or CDC (Centers for Disease Control and Prevention) light traps can be deployed. These methods require either no house entry (exit window traps) or very brief entry by a single entomology team member to clear the traps each day (light traps). PSCs require that house occupants vacate the premises during mosquito collections, enabling appropriate physical distancing. During such visits physical distancing, the wearing of masks and hand sanitisation should be practised. All these methods require home-owner and community engagement and consent.

Garnering support from the community through the establishment of community-based entomological surveillance teams can also be considered. These teams can be trained to collect and appropriately store mosquito larvae and adult mosquitoes, and subsequently submit these specimens to their local malaria control programme personnel.

\section{Information, education and communication, and community sensitisation}

Pre-intervention information, education and communication (IEC) activities, and community sensitisation, are critical components of provincial malaria control programmes, ${ }^{[3,5]}$ to ensure acceptance and uptake of any intervention. It is conceivable and perhaps highly likely that community resistance to malaria control operations, especially IRS and early malaria treatment seeking, will be significant in response to the COVID-19 pandemic. Communities targeted for malaria control during the COVID-19 pandemic should therefore be briefed in detail on the need for malaria control operations to continue uninterrupted. This information sharing can be achieved by adapting IEC messaging, training materials and visuals to include COVID-19 information, while explaining how malaria control operations have been adapted in terms of physical distancing and sanitisation.

Methods for enhanced community IEC and sensitisation include increased media coverage and radio and television slots, and literature in the form of pamphlets, posters and booklets. Launching IRS campaigns through the broadcast media rather than the usual community gatherings should be considered. To establish community 
trust and co-operation, and to maintain momentum, community healthcare workers and environmental health practitioners should conduct robust IEC campaigns. These should be conducted prior to the onset of and during control operations.

Written and verbal messaging should include information on malaria and COVID-19 symptoms, appropriate prevention measures, and advice on healthcare seeking that is adapted to each locality (i.e. advisory and emergency phone numbers and hotlines, and nearest healthcare facilities). Messaging should encourage prompt healthcare seeking should any of the listed symptoms become apparent.

\section{Conclusions and essential recommendations}

Malaria control and elimination operations should continue to be supported at the highest political levels during the ongoing COVID19 pandemic, as encouraged by the WHO. ${ }^{[23]}$ This is important because hard-won gains can easily be eroded should support wane, especially during favourable malaria transmission months/seasons. The adjustments listed provide guidance on how to adapt malaria control and elimination programmes to the COVID-19 situation in SA, Eswatini and southern Mozambique. Based on these, the following essential recommendations are given:

- Healthcare workers must maintain a high index of suspicion for malaria and test for it promptly when patients present with acute febrile illness and other malaria symptoms and signs. All those with positive malaria RDTs (or blood smears) should be started on artemisinin-based therapies immediately, using injectable artesunate for severe malaria and artemether-lumefantrine for uncomplicated malaria.

- Artemisinin-based therapies must only be used to treat malaria; chloroquine and hydroxychloroquine should not be used to treat or prevent COVID-19 (outside of clinical trials).

- Case investigation and patient follow-up practices should continue uninterrupted, but be modified in accordance with COVID-19 prevention guidelines.

- Core vector control activities, specifically IRS and larval source management, should be adapted to accommodate COVID-19 prevention guidelines and conducted timeously and efficiently.

- Vector surveillance activities should continue by utilising outdoorbased collection methods as far as possible, and specific indoor collection methods where indicated, such as the monitoring of indoor-resting mosquitoes in sprayed dwellings.

- Malaria IEC messaging should be adapted to include COVID-19 symptom awareness, prevention and health-seeking advice.

- Community sensitisation campaigns for core malaria control interventions in the context of COVID-19 should be conducted prior to and during operations, especially IRS.

Declaration. None.

Acknowledgements. Goodbye Malaria, a social benefit organisation, in a public-private partnership, aims to eliminate malaria in the MOSASWA region of southern Africa (Mozambique, South Africa, and Eswatini) by 2030.
Author contributions. LB and KR initialised the review and contributed to various sections. BDB drafted certain sections, collated input provided by the other authors and produced the final version of the manuscript. JR and JF wrote the initial drafts to certain sections and contributed to others, and assisted with editing. FM, EM, AM, KIB, DPM, QD and SC contributed to specific sections. LB approved the final version.

Funding. None.

Conflicts of interest. None.

1. Giandhari J, Pillay S, Wilkinson E, et al. Early transmission of SARS-CoV-2 in South Africa: An epidemiological and phylogenetic report. medRxiv 2020. https://doi.org/10.1101/2020.05.29.20116376 2. Raman J, Morris N, Frean J, et al. Reviewing South Africa’s malaria elimination strategy (2012 - 2018): Progress, challenges and priorities. Malar J 2016;15(1):438. https://doi.org/10.1186/s12936-016-1497-x 3. National Department of Health, South Africa. Malaria elimination strategic plan for South Africa 2019 - 2023. 2019. http://www.health.gov.za/index.php/publications?download=3571:malaria-eliminationstrategic-plan-south-africa-2019-2023 (accessed 10 August 2020).

4. World Health Organization. Malaria elimination by 2020 is possible in Swaziland. 7 June 2017. https:// wrwwafrowho int/news/malaria-elimination-2020-possible-swaziland (accessed 10 Augut 2020 ).

Www.afro.who.int/news/malaria-elimination-2020-possible-swailand (accessed 10 August 2020).

Moonasar D, Maharaj R, Kunene S, et al. Towards malaria elimination in the MOSASWA
(Mozambique, South Africa and Swaziland) region. Malar J 2016;15(1):419. https://doi.org/10.1186/ s12936-016-1470-8

6. World Health Organization. World Malaria Report 2019. 4 December 2019. https://www.who.int/ publications/i/item/world-malaria-report-2019 (accessed 10 August 2020).

7. Aide $P$, Candrinho B, Galatas B, et al. Setting the scene and generating evidence for malaria elimination in southern Mozambique. Malar J 2019;18(1):190. https://doi.org/10.1186/s12936-019-2832-9

8. Parpia AS, Ndeffo-Mbah ML, Wenzel NS, Galvani AP. Effects of response to 2014 - 2015 Ebola outbreak on deaths from malaria, HIV/AIDS, and tuberculosis, West Africa. Emerg Infect Dis 2016:22(3):433441. https://doi.org/10.3201/eid2203.150977

9. Davies C, Graffy R, Shandukani M, et al. Effectiveness of 24-h mobile reporting tool during a malaria

Davies C, Graffy R, Shandukani M, et al. Effectiveness of 24-h mobile reporting tool during a malaria
outbreak in Mpumalanga Province, South Africa. Malar J 2019;18(1):45. https://doi.org/10.1186/ outbreak in Mpum
s12936-019-2683-4

10. Dittrich S, Lamy M, Acharya S, et al. Diagnosing malaria and other febrile illnesses during the COVID-19 pandemic. Lancet Glob Health 2020;8(7):e879-e880. https://doi.org/10.1016//2214109X(20)30210-2

11. Rosenthal PJ, Breman JG, Djimde AA, et al. COVID-19: Shining the light on Africa. Am J Trop Med Hyg 2020;102(6):1145-1148. https://doi.org/10.4269/ajtmh.20-0380

12. Porte L, Legarraga $P$, Vollrath $V$, et al. Evaluation of novel antigen-based rapid detection test for the diagnosis of SARS-CoV-2 in respiratory samples. Int J Infect Dis 2020;99:328-333. https://doi. org/10.1016/j.ijid.2020.05.098

13. Di Gennaro F, Marotta C, Locantore P, Pizzol D, Putoto G. Malaria and COVID-19: Common and Di Gennaro F, Marotta C, Locantore P, Pizzol D, Putoto G. Malaria and COVID-19: Common and
different findings. Trop Med Infect Dis 2020;5(3):141. https://doi.org/10.3390/tropicalmed5030141

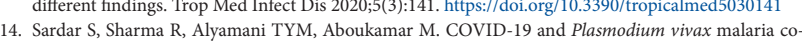
4. Sardar S, Sharma R, Alyamani TYM, Aboukamar M. COVID-19 and Plasmo
infection. IDCases 2020;21:e00879. https://doi.org/10.1016/j.idcr.2020.e00879

15. World Health Organization. Guidelines for the Treatment of Malaria. 3rd ed. Geneva: WHO, 2015. https://apps.who.int/iris/bitstream/handle/10665/162441/9789241549127_eng.pdf (accessed 15 August 2020).

16. Sinclair D, Donegan S, Isba R, Lalloo DG. Artesunate versus quinine for treating severe malaria Cochrane Database Syst Rev 2012, Issue 6. Art. No.: CD005967. https://doi.org/10.1002/14651858. CD005967.pub4

17. Skipper CP, Pastick KA, Engen NW, et al. Hydroxychloroquine in non-hospitalised adults with early COVID-19: A randomised trial. Ann Intern Med 2020 (e-pub 16 July 2020). https://doi.org/10.7326/
Cority COVID-19: A

18. Boulware DR, Pullen MF, Bangdiwala AS, et al. A randomised trial of hydroxychloroquine as postexposure prophylaxis for COVID-19. N Engl J Med 2020;383(6):517-525. https://doi.org/10.1056 NEJMoa2016638

19. Schulger NW. The saga of hydroxychloroquine and COVID-19: A cautionary tale. Ann Intern Med 2020 (epub 16 July 2020). https://doi.org/10.7326/M20-5041

20. Michaud V, Dow P, Al Rihani SB, et al. Risk of drug-induced long QT syndrome associated with the use of repurposed COVID-19 drug: A systematic review. medRxiv 2020 (epub 2 September 2020). https:// doi.org/10.1101/2020.04.21.20066761

21. World Health Organization. Guidelines for malaria vector control. February 2019. https://www.who. World Health Organization. Guidelines for malaria vector control. February
int/malaria/publications/atoz/9789241550499/en/ (accessed 15 August 2020).

22. Dandalo LC, Brooke BD, Munhenga G, et al. Population dynamics and Plasmodium falciparum (Haemosporida: Plasmodiidae) infectivity rates for the malaria vector Anopheles arabiensis (Diptera Culicidae) at Mamfene, KwaZulu-Natal, South Africa. J Med Entomol 2017;54(6):1758-1766. https:// doi.org/10.1093/jme/tjx169

23. World Health Organization. Tailoring malaria interventions in the COVID-19 response. 9 April 2020. https://www.who.int/publications/m/item/tailoring-malaria-interventions-in-the-covid-19-response (accessed 15 August 2020)

Accepted 6 October 2020 